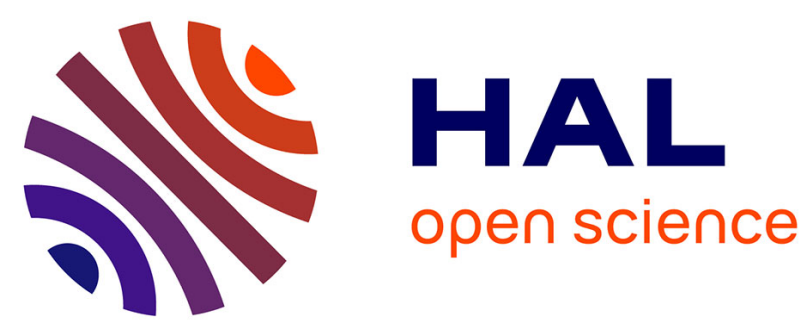

\title{
Recherches sur les biocénoses céréalières II. - Incidence des interventions insecticides sur les composants de l'entomofaune
}

Jean-Pierre Chambon, Gilles Genestier, Michel Martinez, Colette Pineau, Christian Cocquempot, Jean-Louis Coste, Daniel Serre

\section{To cite this version:}

Jean-Pierre Chambon, Gilles Genestier, Michel Martinez, Colette Pineau, Christian Cocquempot, et al.. Recherches sur les biocénoses céréalières II. - Incidence des interventions insecticides sur les composants de l'entomofaune. Agronomie, 1982, 2 (5), pp.405-416. hal-00884399

\section{HAL Id: hal-00884399 https://hal.science/hal-00884399}

Submitted on 1 Jan 1982

HAL is a multi-disciplinary open access archive for the deposit and dissemination of scientific research documents, whether they are published or not. The documents may come from teaching and research institutions in France or abroad, or from public or private research centers.
L'archive ouverte pluridisciplinaire HAL, est destinée au dépôt et à la diffusion de documents scientifiques de niveau recherche, publiés ou non, émanant des établissements d'enseignement et de recherche français ou étrangers, des laboratoires publics ou privés. 


\title{
Recherches sur les biocénoses céréalières \\ II. - Incidence des interventions insecticides sur les composants de l'entomofaune
}

\author{
Jean-Pierre CHAMBON \\ avec la collaboration technique de Gilles GENESTIER, Michel MARTINEz, Colette PINEAU, Christian \\ COCQUEMPOT, Jcan-Louis COSTE $(*)$ \& Danicl SERRE $(* *)$ \\ I.N.R.A., Station de Zoologie, Laboratoire de Faunistique écologique Route de St. Cyr, F 78000 Versailles. \\ (*) I.T.C.F., F 91920 Boigneville. \\ (**) O.N.C., F 28024 Chartres.
}

\section{RÉSUMÉ}

Biocénose, Entomofaune.

Céréales.

Effets secondaires,

Insecticides,

Ravageurs.

La systématisation et la généralisation des traitements phytosanitaires peuvent avoir des répercussions secondaires indésirables et graves sur les équilibres fauniques déjà précaires de ces milieux artificiels que sont les agrosystèmes céréaliers qui représentent en France 9000000 ha en 1980.

Nous avons engagé, en 1978 et 1979, des recherches sur l'action de traitements insecticides (parathion méthyl + phosalone et diméthoate) en végćtation sur l'entomofaune des champs de blé.

Si les niveaux de populations d'insectes sont très bas dans les régions traitées intensément depuis plusieurs années, ce sont les espèces nuisibles qui occupent une place prépondérante.

Les captures journalières accusent une chute de l'ordre de 60 à 80 p. 100 après traitement. La faune détruite représente, à cette période, en majeure partie des insectes utiles. L'analyse du matériel capturé avant et après traitement montre que les groupes les plus touchés sont les Carabidae, les Staphylinidae, les Collemboles, les Hyménoptères. Les captures augmentent à nouveau après 12 à 15 jours.

La recolonisation par l'entomofaune, constatée actuellement, n'est possible que grâce à la faible proportion des surfaces traitées.

Une évolution dans le sens de l'intensification et la généralisation des interventions avec des produits à large champ d'activité ne pourrait qu'aggraver la situation et déboucher sur de nouveaux problèmes phytosanitaires.

\section{SUMMARY}

Biocenosis,

Entomofauna,

Cereals,

Side-effects.

Insecticides,

Pests.
Studies on the cereal agroecosystem - II. - Side-effects of insecticide treatments on the entomofauna

We have attracted growers' attention to the fact that insecticide treatments can have major side-effects on the faunal balance.

This risk is increased by the size of the areas concerned ( 9000000 ha of cereals in France), and by the more regular and general use of these treatments.

This is the reason why we have put forward the idea of the "slice of life " which can be killed by treatments. For this reason, we started research in 1978-79 on the effect of insecticide treatments on the entomofauna during cereal growth.

Two insecticides : methylparathion + phosalone and dimethoate were used at normal application rates.

It appears in the first instance, that in heavily treated areas, after several years, the level of insect populations are very low, but pest species become more numerous. Comparison of daily catches before and after treatment shows that catches decreased about $60-70 \%$ after treatment with dimethoate and about $70-80 \%$ with methyl parathion + phosalone. Since, according to our observations, there was very few pests at this period. The killed fauna must have been constituted for the greater part of useful insects in the widest sense of the word.

Analysis of catches before and after treatment confirms this, the most affected groups being Carabidae. Staphylinidae. Collembola, Hymenoptera.

Catches increased again after 12-15 days, possibly because of the small percentage of treated areas.

Intensification and generalization of treatments with a limited number of specific pesticides will aggravate the situation and give new problems in plant protection.

\section{INTRODUCTION}

A la suite de plusieurs années d'observations effectuées sur les biocénoses céréalières dans la région de Fontainc- bleau : La Chapelle-La-Reine et Ury (77) et Dadonville (45) (CHAMBON. 1982) où les interventions phytosanitaires en cours de végétation n'ont pas encore pris un développcment important, nous avons mis en évidence la complexité 
et la richesse de l'entomofaune présente au cours de la période de végétation. Cette faune est composée pour $95 \mathrm{p}$. 100 d'espèces auxiliaires et détritiphages, dont le rôle est important dans le fonctionnement de ces agrosystèmes, et, pour 5 p. 100 , d'espèces phytophages nuisibles.

A partir de ces premières constatations, nous avons rapidement attiré l'attention des praticiens sur le fait que les interventions phytosanitaires (fongicides, herbicides, insecticides) dont l'action n'est pas aussi étroite que leur dénomination le laisserait sous-entendre, pouvaient avoir des répcrcussions secondaires indésirables et graves sur les équilibres fauniques déjà précaires de ces milieux artificiels que sont les agrosystèmes. Ce risque est encore accru par l'importance des surfaces concernées $(9000000$ ha de céréales en France en 1980), la systématisation et la généralisation de ces pratiques.

C'est ainsi que nous avons mis en avant la notion de "tranches de vie" (CHAMBON, 1975) qui pouvaicnt être détruites. Dans cette optiquc, nous avons engagé cn 1978 et en 1979 des recherches sur l'action des traitements insecticides en végétation, sur l'entomofaune des champs de céréales à paille (blé).

L'objectif de ces recherches est de préciser l'importance et la qualité de la faune détruite à la suite des traitements insecticides et d'en analyser les conséquences pratiques à long terme.

\section{INCIDENCES DES INTERVENTIONS PHYTOSA- NITAIRES SUR LES COMPOSANTS DES BIOCÉNO- SES CÉRÉALIËRES}

\section{A. Expérimentation 1978}

\section{Conditions d'expérimentation}

Ces études menées en collaboration avec l'ITCF ont été réalisées dans deux champs:

- l'un à la Chapelle-la-Reine (LCLR) en Seine-etMarne, recevant seulement un herbicide;

- l'autre à Bretonvilliers (BRET) en Eure-et-Loir, recevant l'ensemble des interventions susceptibles d'assurer une protection aussi complète que possible vis-à-vis des 2 ravageurs potentiellement présents dans la région (Cnephasia pumicana $Z$. et Sitobion avenae Fabre). Cette culture se trouve dans une zone où des traitements sont pratiqués depuis plusicurs années de manière courante, à savoir : 4 interventions annuelles dont 2 avec des insecticides, les matières actives étant utilisées aux doses habituelles d'cmploi.

a) Traitements en végétation appliqués en 1978.

- la Chapelle-la-Reine : le 18 avril, herbicide $(2,4-\mathrm{D}+2,4$ M(PA) ;

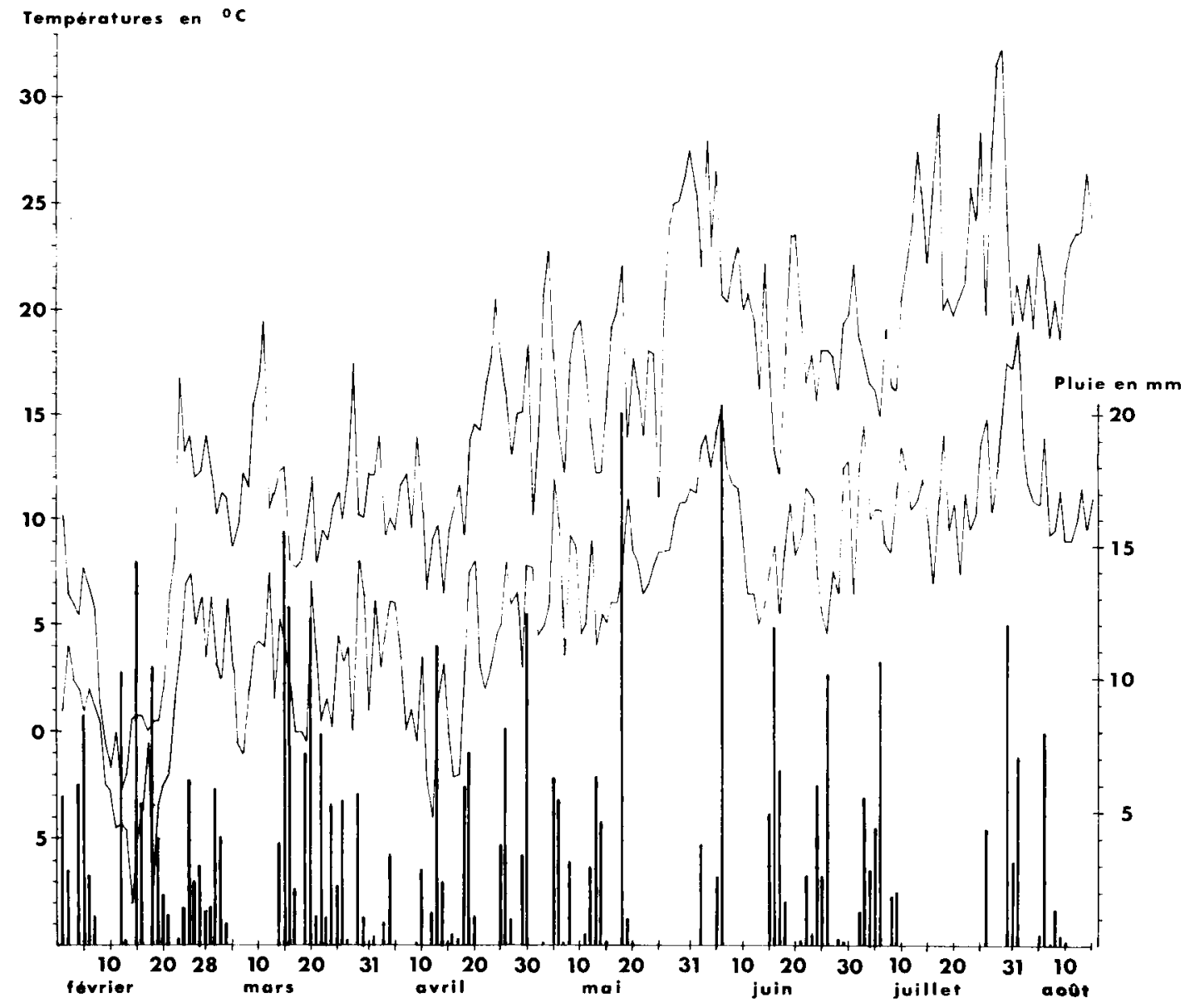

Figurc 1

Relevé des conditions climatiques en 1978 à La Chapelle-la-Reine.

Climatic conditions at la Chapelle-la-Reine in 1978. 


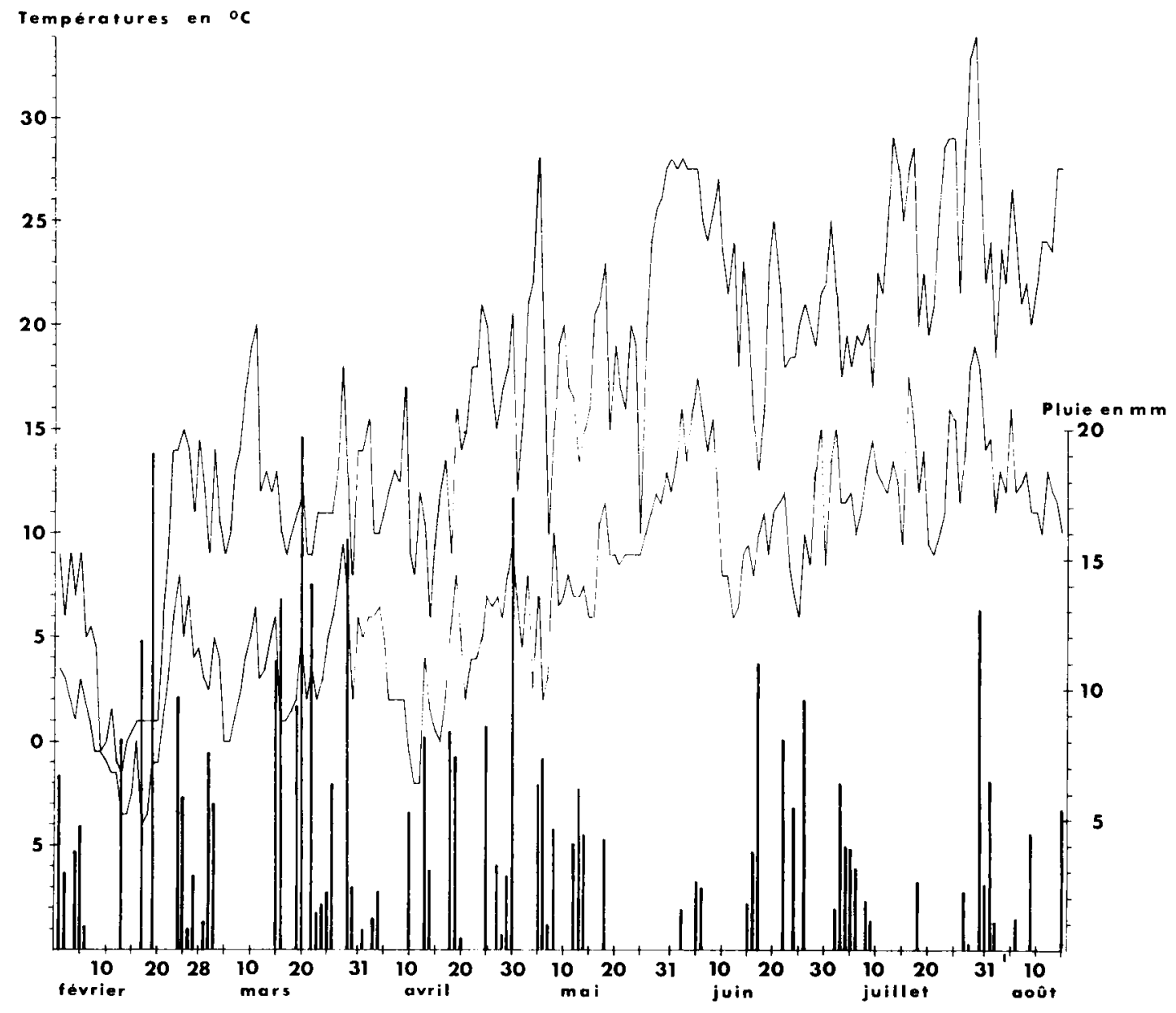

Figure 2

Relevé des conditions climatiques en 1978 à Bretonvilliers.

Climatic conditions at Bretonvilliers in 1978.

- Bretonvilliers :

le 7 avril, herbicide (isoproturon + dinoterbe), le 10 mai, fongicide (carbendazime + mancozèbe), le 16 mai, herbicide (2,4-D), insecticide (parathion), le 8 juin, fongicide (carbendazime + triadiméfon), insecticide (diméthoate).

b) Conditions climatiques.

Relevées quotidiennement de début février à mi-août, les températures et la pluviométrie à LCLR et BRET sont sensiblement voisines comme le montrent les figures 1 et 2 .

\section{c) Technique d'étude.}

Dans chaque champ, nous avons utilisé les mêmes techniques de piégeage, le même dispositif et la même périodicité des relevés que pour l'étude rapportée dans la $1^{\text {rc }}$ partic.

\section{Résultats de l'étude}

L'analyse du matériel récolté fait apparaitre les données regroupées dans le tableau 1.

Les méligèthes étant, de toutc ćvidence, étrangers à la culture et uniquement des insectes de passage sur lesquels les traitements antérieurs à lcur arrivée n'ont pas d'incidence, il nous a paru judicieux de ne pas en tenir compte dans nos interprétations.
On peut noter que le nombre d'espèces recensées est plus élevé à LCLR qu'à BRET, ceci traduisant l'existence d'une faune plus diversifiée.

On constate aussi que l'essentiel des captures (97,5 p. 100 dans les 2 champs) est constituć par les 180 espèces communes déjà recensées (CHAMBON, 1982) montrant ainsi que les peuplements ne sont pas fondamentalement différents. Ceci nous autorise à ne pas prendre en considération les espèces particulières qui représentent dans chaque cas 2,5 p. 100 environ des captures et dont les effectifs individuels sont très faibles.

Par aillcurs, la lecture du tableau 1 fait apparaître d'emblée que, dans ces 2 blés sur précédents maïs, les captures globales sont très différentes en effectifs. En cffet, nous avons 43350 individus (soit $44,7 \mathrm{p}$. 100) de plus à LCLR qu'à BRET. Il apparaît donc que, pour les mêmes cspèces, les populations sont nettement inférieures à BRET. Rappelons que ces cultures se différencient par le nombre des traitements appliqués en végétation. De surcroît, elles sont situées dans des environnements phytosanitaires différents, ainsi LCLR est dans une zone très peu traitéc depuis des années, alors que BRET est dans une zone traitéc de façon plus intense et plus régulière depuis 1974.

Il faut remarquer que les captures d'insectes ravageurs sont inférieures à LCLR où ils ne représentent que 11 p. 100 contre 22 p. 100 à BRET. Il semble donc que les popula- 
TABLEAU 1

Analyse du matériel récolté en 1978.

Analysis of material collected in 1978.

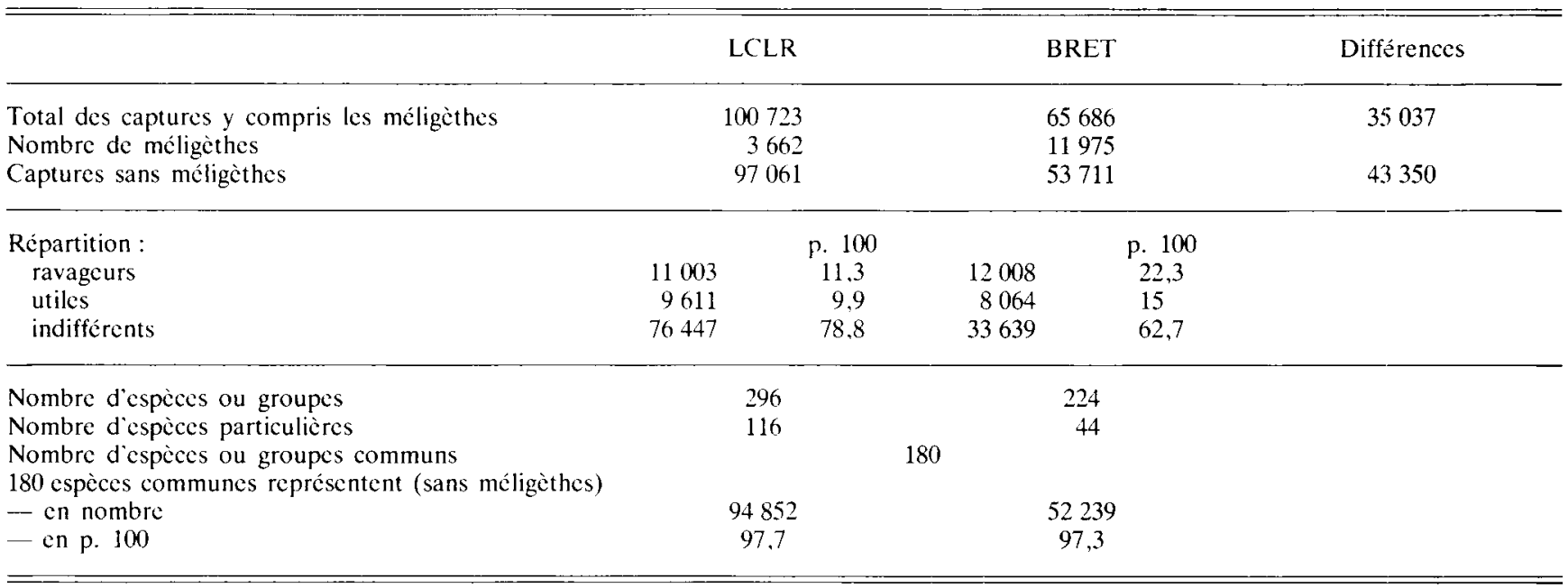

tions de nuisibles ont une importance relative supéricure à celle observée dans le champ de la zone non traitée.

Cette $1^{\text {re }}$ analyse de la situation générale nous donne déjà un aperçu de l'ćvolution des populations d'insectes en zone traitée.

Le $2^{c}$ objectif de notre étude était d'apprécier l'action directe du traitement insecticide sur la faune du champ en notant les captures hebdomadaires avant et après l'intervention et en les comparant à celles enregistrées à LCLR (non traitée).

Une forte pluviométric, perturbant les captures et masquant l'effet du traitement, associée à une périodicité des relevés de captures trop espacée permettant, compte tenu du potenticl de déplacement des insectes, une recolonisation atténuant les chutes des populations, n'ont pas permis d'établir cette comparaison. Aussi avons nous repris l'expérimentation en 1979 en effectuant des relevés journaliers. Néanmoins, les relevés de 1978 nous ont montré plusieurs résultats significatifs dont nous présentons 2 exemples se rapportant à des coléoptères prédatcurs.

- Pour Anchomenus dorsalis Pont. (fig. 3), on voit que les captures sont beaucoup plus faibles à BRET qu'à LCLR, ce qui confirme les remarques faites précédemment sur les niveaux de populations. Les traitements des 10 et 16 mai freinent le développement de la population, contrairement à LCLR où nous avons une augmentation des captures. De plus, l'intervention du 8 juin se traduit par un arrêt net des prises à BRET dès le 12 juin, alors qu'à LCLR celles-ci sont encore très abondantes jusqu'au 3 juillet.

- Pour les Staphylinidae (fig. 4), nous avons une évolution parallèle dans les 2 champs jusqu'au 8 mai, puis une inversion à BRET qui marque l'action des traitements des 10 et 16 mai. De la même façon, après l'intervention du 8 juin, on note unc chute à BRET alors que les captures augmentent à LCLR.

\section{B. Expérimentation 1979}

En 1979, nos recherches ont été poursuivies dans 3 champs situćs en Eure-et-Loir : 1 à Bretonvilliers (BRET), 2 à Louville-la-Chenard $\left(\mathrm{L}_{1}, \mathrm{~L}_{2}\right)$, que nous avons comparés à un $4^{c}$ non traité, sis à Ury (Seine-et-Marne).

Le dispositif expérimental mis en place est le même qu'en 1978. Seul change le rythme des prélèvements qui devient quotidien au cours des semaines précédant et suivant le traitement.

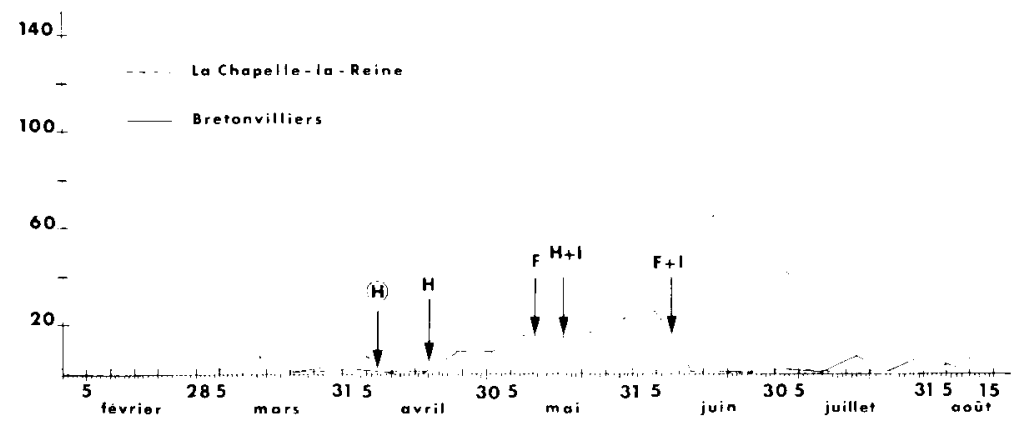




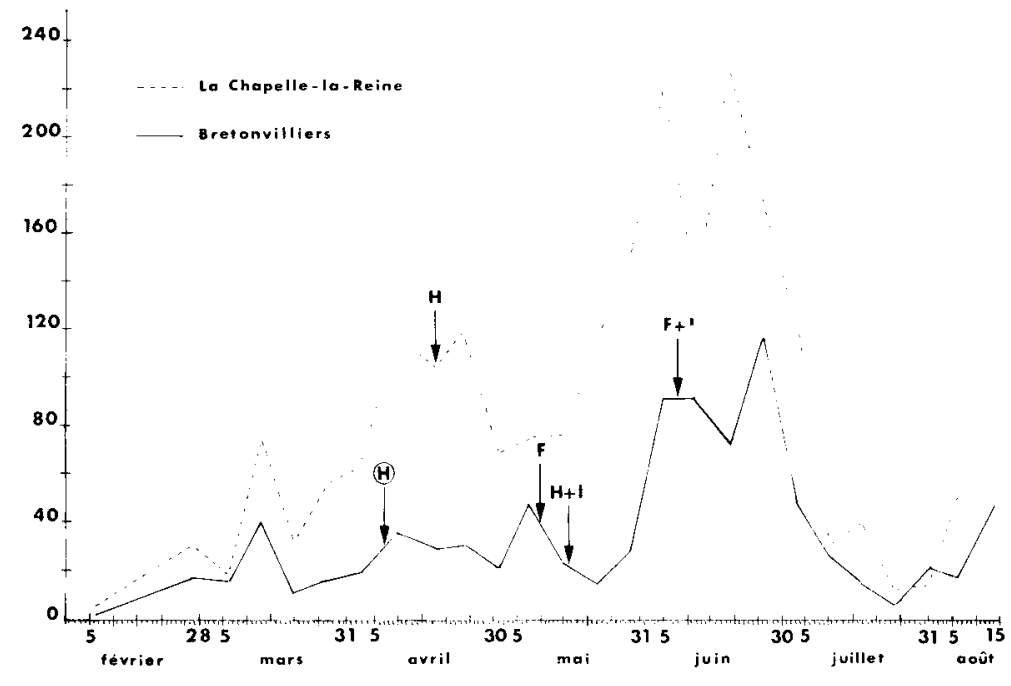

Figure 4

Evolution des captures de Staphylinidae

$H$ : herbicide : colorants nitrés,

$H$ : herbicide: hormone végétale,

$F$ : fongicide,

$I$ : insecticide,

$\downarrow$ : traitements.

Catches of Staphylinidae

$H$ : herbicides : phenols,

$H$ : herbicides : phenoxyacetic acids,

$F$ : fongicide

I: insecticide,

$\downarrow$ : treatment

\section{Bretonvilliers}

\section{a) Conditions d'expérimentation}

Les données climatiques sont regroupées dans les figures 5 et 6 .

Les traitements reçus par le champ sont :

- le 12 mai, herbicide (dichlorprop + 2,4-MCPA + mécoprop), fongicide (carbendazime + mancozèbe), insecticide (parathion méthyl) ;

- le 13 juin, fongicide (carbendazime + mancozèbe), insecticide (diméthoate).

\section{b) Résultats}

Les captures globales sont regroupées dans le tableau 2. Ces résultats confirment ceux obtenus en 1978, à savoir que les niveaux de population sont très bas à BRET. Les collemboles, en particulier, constituent l'essentiel de la différence.

Dans ces conditions, les baisses des niveaux de captures notées après le traitement du 12 mai sont de 4 p. 100 en pièges colorés $(\mathrm{PC})$ et de 9 p. 100 en pots-pièges $(\mathrm{PP})$; elles se situent à 49 p. 100 en PC et 11 p. 100 en PP après le traitement du 13 juin.

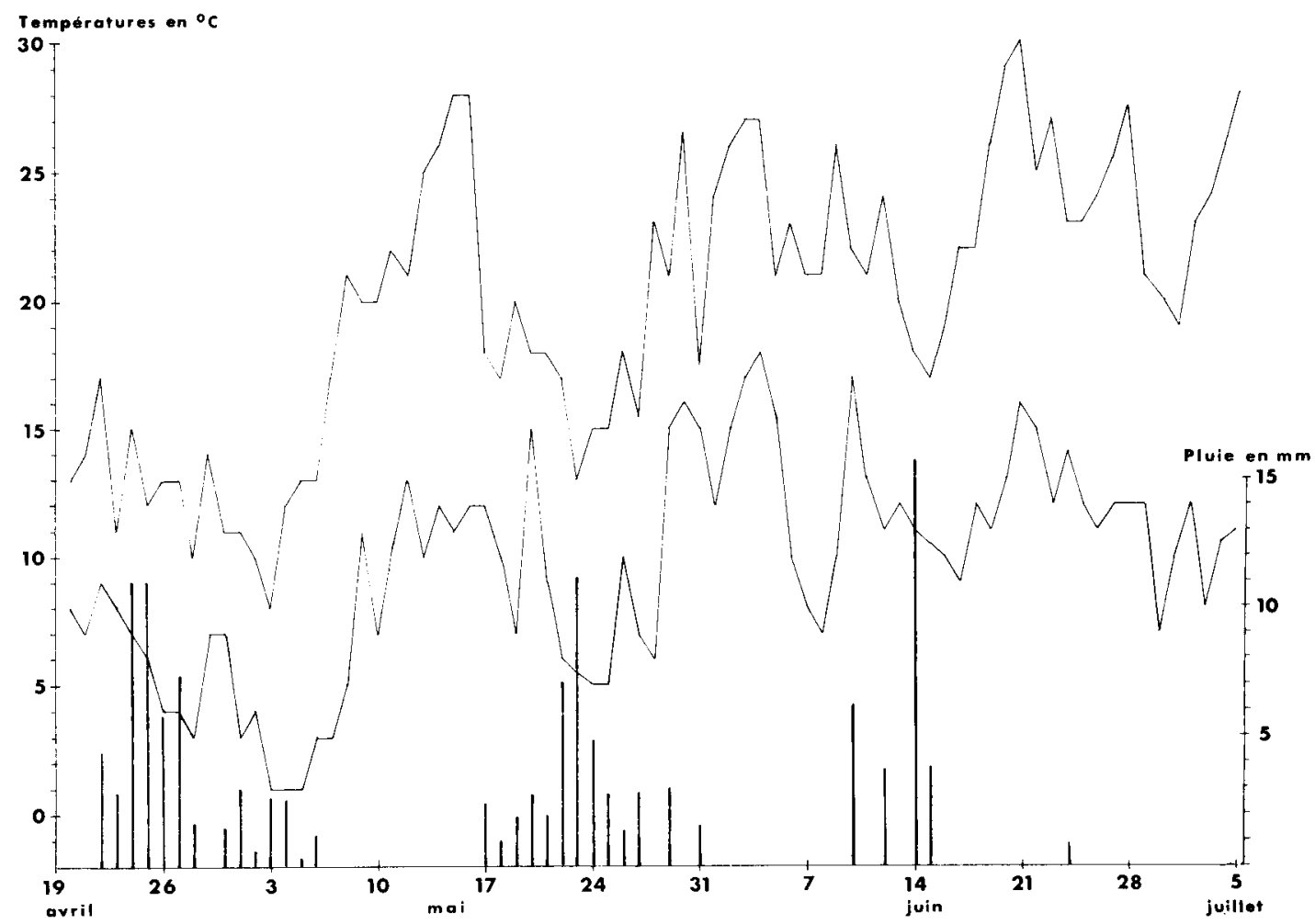

Figure 5

Conditions climatiques en 1979 à Bretonvilliers.

Climatic conditions at Bretonvilliers in 1979. 


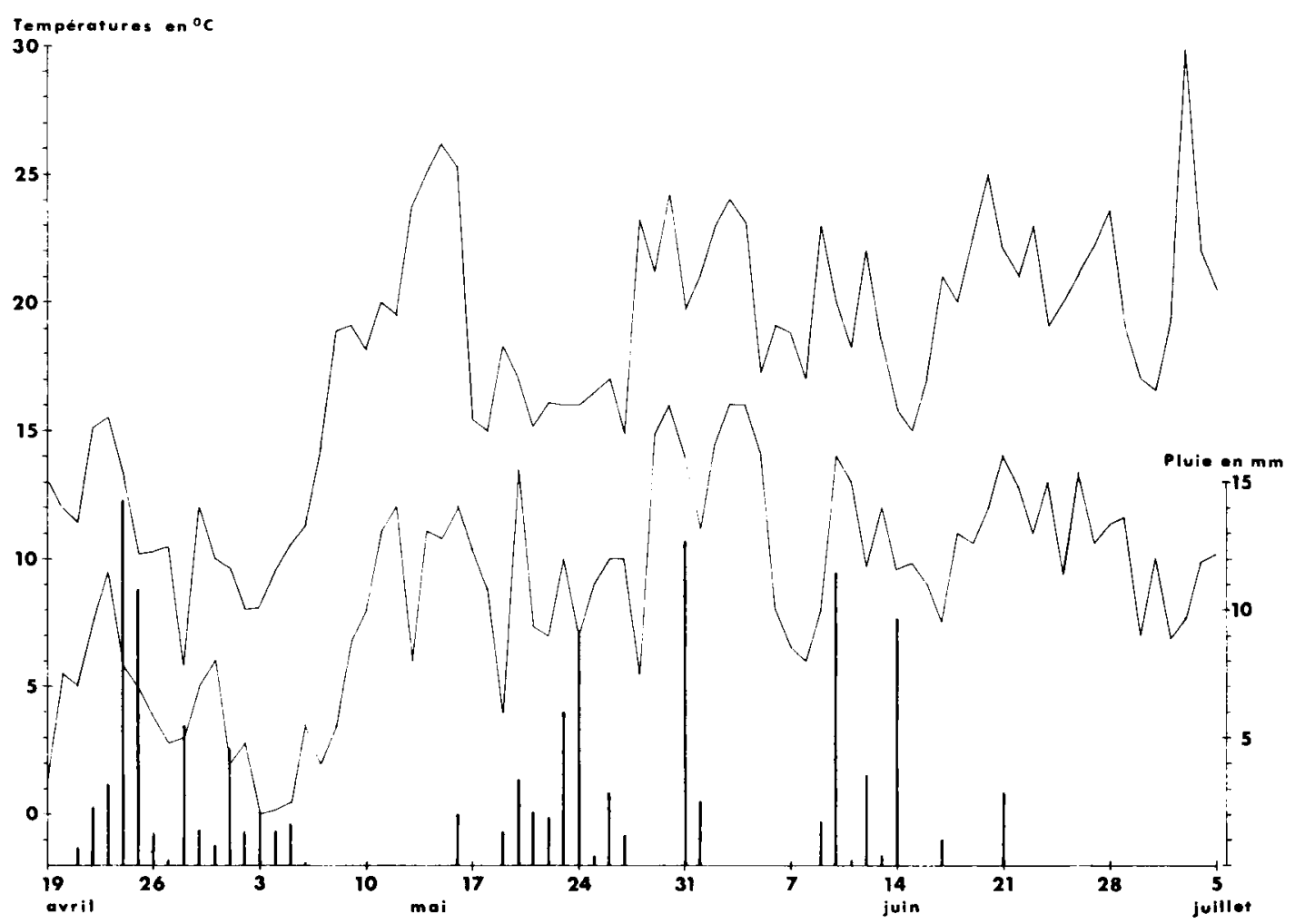

Figure 6

Conditions climatiques en 1979 à Ury.

Climatic conditions at Ury in 1979.

Ces chutes relativement faibles peuvent s'expliquer par les faibles effectifs capturés à BRET, liés à des niveaux de population très bas qui masquent en partie l'action brutale de l'intervention, surtout si on émet l'hypothèse que les populations résiduelles sont constituées pour une grande part d'individus peu sensibles aux produits utilisés à la suite de plusieurs campagnes de traitements en cours de végétation.

\section{Louville-la-Chenard}

\section{a) Conditions d'expérimentation}

Deux champs de blé $\left(\mathrm{L}_{1}, \mathrm{~L}_{2}\right)$ situés dans un secteur peu traité ont été suivis ; dans chaque champ, on place une unité de piégeage à $30 \mathrm{~m}$ de toute bordure (IB) et une autre en lisière d'un chemin enherbé (BC).
Le champ $\mathrm{L}_{1}$ est traité le 19 juin avec du diméthoate.

Le champ $L_{2}$ est traité le 12 juin avec une spécialité commerciale associant le phosalone et le parathion-méthyl.

Les conditions climatiques de la période située entre le 15 mai et le 15 juillet sont regroupées à la figure 7 .

\section{b) Résultats}

a) Mise en évidence de l'action globale du traitement

Pour faciliter la lecture et l'interprétation des données, nous les avons regroupées dans les tableaux 3,4 et 5 . La lecture du tableau 4 montre qu'il y a une baisse importante des niveaux de captures après les traitements : de 60 à $70 \mathrm{p}$. 100 dans $L_{1}$ avec le diméthoate et de 70 à $80 \mathrm{p} .100$ avec l'association phosalone + parathion-méthyl dans $\mathrm{L}_{2}$.

Il était évidemment nécessaire de s'assurer que les différences observées n'étaient pas naturelles, dues au cycle

TABLEAU 2

Captures globales à BRET (1979). PP: pots pièges; $P C$ : plateaux colorés.

Total catches at BRET in 1979. PP : pitfall traps; PC: yellow traps.

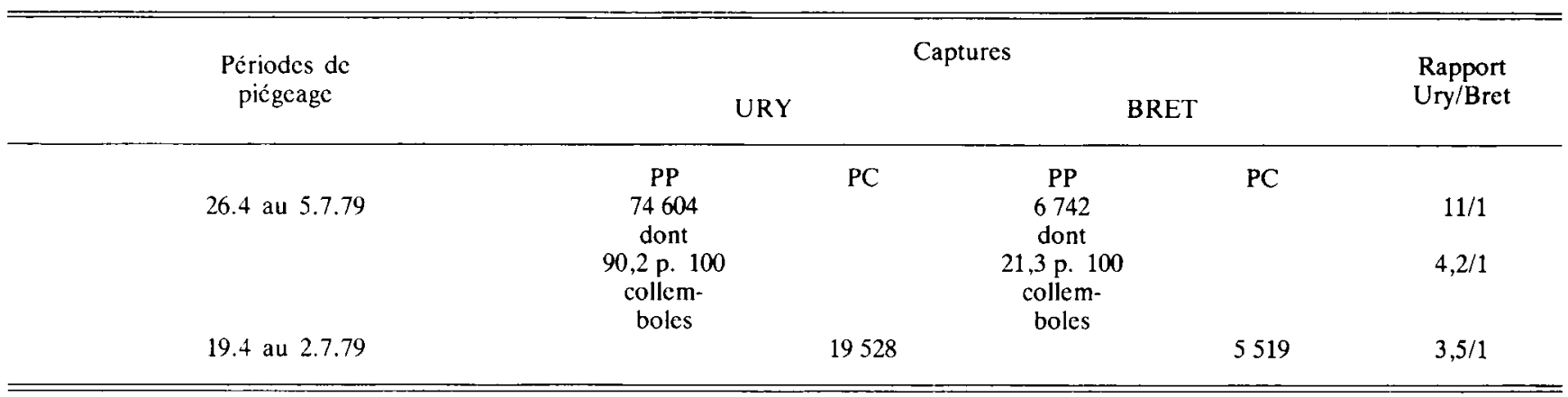




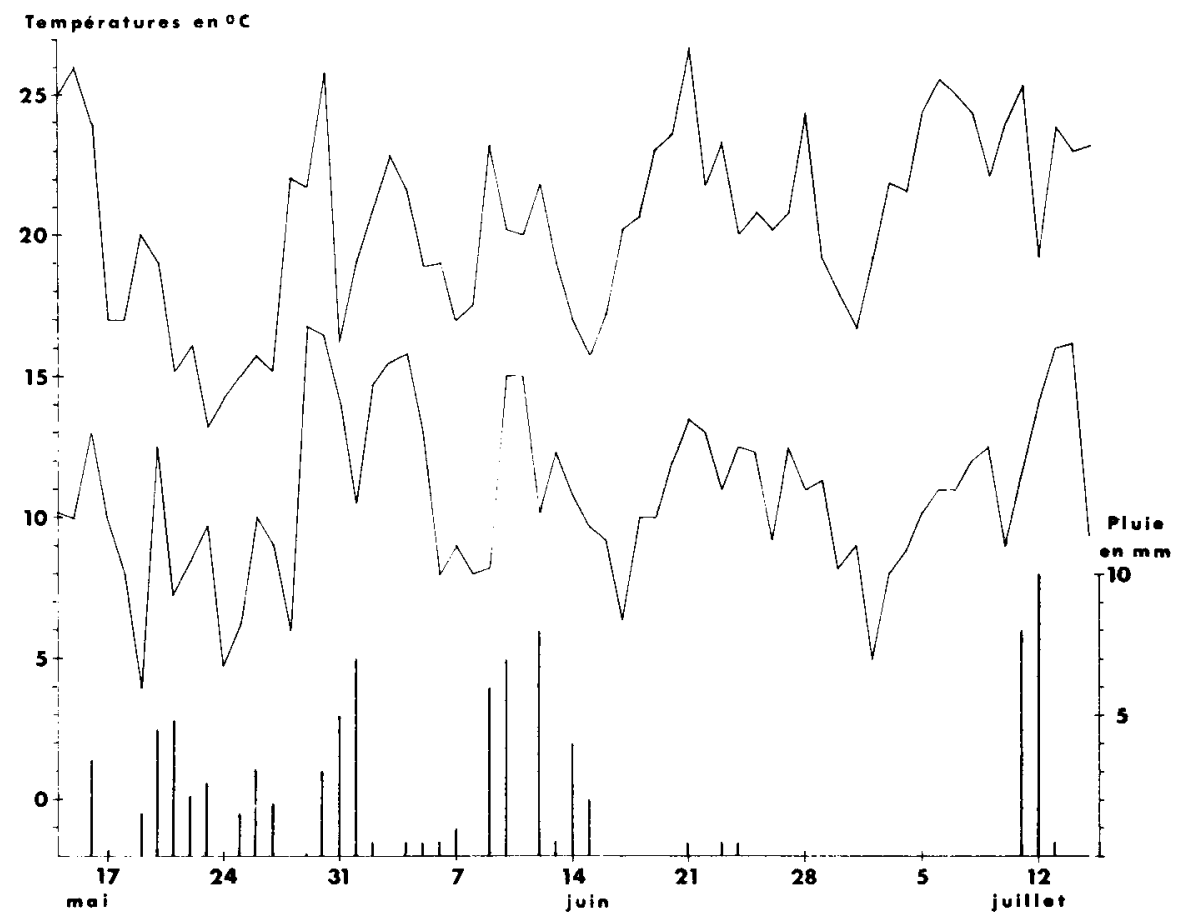

Figure 7

Conditions climatiques en 1979 à Louville-la-Chenard.

Climatic conditions at Louville-la-Chenard in 1979.

TABLEAU 3

Captures globales à $L_{1} L_{2}$ (1979). PP : pots pièges; $P C$ : plateaux colorés; $I B$ : intérieur du champ; $B C$ : bordure du champ.

Total catches at $L_{1} L_{2}$ in 1979. PP : pitfall traps; $P C$ : yellow traps; $I B$ : inside the field; $B C:$ on the edge of the field.

\begin{tabular}{|c|c|c|c|c|c|c|c|c|}
\hline \multirow{3}{*}{$\begin{array}{l}\text { Période de } \\
\text { piégeage }\end{array}$} & \multicolumn{8}{|c|}{ Total des captures } \\
\hline & \multicolumn{2}{|c|}{ PP } & \multicolumn{2}{|c|}{$\mathrm{PC}$} & \multicolumn{2}{|c|}{ PP } & \multicolumn{2}{|c|}{$\mathrm{PC}$} \\
\hline & IB & $\mathrm{BC}$ & IB & $\mathrm{BC}$ & IB & $\mathrm{BC}$ & IB & $\mathrm{BC}$ \\
\hline $\begin{array}{c}4.6 \text { au } 5.7 \\
21.5 \text { au } 16.7 \\
21.5 \text { au } 12.7 \\
17.5 \text { au } 16.7\end{array}$ & 18865 & 18935 & 12669 & 15459 & 17125 & 13638 & 10943 & 12901 \\
\hline
\end{tabular}

\section{TABLEAU 4}

Incidences immédiates des traitements sur les captures dans les champs $L_{1} L_{2}$. PP : pots pièges ; $P C$ : plateaux colorés; IB : intérieur du champ ; $B C$ : bordure du champ.

Immediat effects of sprays on catches in field $L_{1} L_{2} . P P$ : pitfall traps;

$P C$ : yellow traps; $I B$ : inside the field; $B C$ : on the edge of the field.

$\mathbf{L}_{1}$
PC
$\mathrm{L}_{2}$

\begin{tabular}{|c|c|c|c|c|c|c|c|c|}
\hline & \multicolumn{2}{|c|}{ PP } & \multicolumn{2}{|c|}{$\mathrm{PC}$} & \multicolumn{2}{|c|}{ PP } & \multicolumn{2}{|c|}{ PC } \\
\hline & IB & $\mathrm{BC}$ & IB & $\mathrm{BC}$ & IB & $\mathrm{BC}$ & IB & $\mathrm{BC}$ \\
\hline \multirow{5}{*}{$\begin{array}{l}\text { Nombre de captures } \\
\text { avant traitement } \\
\mathrm{m} / \mathrm{J} \\
\text { Nombre de captures } \\
\text { après traitement } \\
\mathrm{m} / \mathrm{J} \\
\text { Baisse en p. } 100\end{array}$} & $5708 / 8 \mathrm{~J}$ & $5957 / 8 \mathrm{~J}$ & $3692 / 8 \mathrm{~J}$ & $2954 / 8 J$ & $7987 / 8 \mathrm{~J}$ & $10790 / 13 \mathrm{~J}$ & $2411 / 5 \mathrm{~J}$ & $7463 / 13 \mathrm{~J}$ \\
\hline & 713 & 744 & 461 & 369 & 998 & 830 & 482 & 574 \\
\hline & $2871 / 9 \mathrm{~J}$ & $2715 / 9 \mathrm{~J}$ & $1251 / 9 \mathrm{~J}$ & $1049 / 9 \mathrm{~J}$ & $1421 / 8 \mathrm{~J}$ & $1939 / 13 \mathrm{~J}$ & $660 / 5 \mathrm{~J}$ & $1647 / 13 \mathrm{~J}$ \\
\hline & 319 & 301 & 139 & 116 & 177 & 149 & 132 & 126 \\
\hline & 56 & 60 & 70 & 69 & 83 & 83 & 73 & 79 \\
\hline
\end{tabular}




\section{TABLEAU 5}

Comparaison des captures dans les champs $L_{1} L_{2}$ (traités) avec celles du champ Ury (non traité). IB : intérieur du champ; PP : pots pièges ; PC : plateaux colorés.

Comparison of catches in the $L_{1} L_{2}$ treated fields and the Ury untreated field. IB: inside the field; PP: pitfall traps; PC: yellow traps.

\begin{tabular}{|c|c|c|c|}
\hline \multirow{2}{*}{ Champs } & \multicolumn{3}{|c|}{ Pćriodes de captures } \\
\hline & du 7.6 au 14.6 & du 14.6 au 21.6 & 21.6 au 28.6 \\
\hline URY IBPP & 4881 & 6257 & 4755 \\
\hline $\mathrm{L}_{1}$ IBPP & 6973 & $4052\left(^{*}\right)$ & 1296 \\
\hline $\mathrm{L}_{2}$ IBPP & $5441(* *)$ & 585 & 640 \\
\hline URY IBPC & 1918 & 1235 & 2030 \\
\hline $\mathrm{L}_{1}$ IBPC & 2494 & $1229\left(^{*}\right)$ & 605 \\
\hline$L_{2}$ IBPC & $3426(* *)$ & 618 & 467 \\
\hline
\end{tabular}

$\left(^{*}\right)$ Traitement du 19.6

$\left({ }^{*}\right)$ Spray on 19.VI.

(**) Traitement du 12.6.

(**) Spray the 12 .VI.

évolutif des insectes ou liées à de fortes précipitations ou bien encore au stade phénologique des cultures.

L'examen des relevés pluviométriques fait ressortir qu'avant les traitements, on a un nombre de jours de pluie et des quantités d'eau tombée supérieurs à ceux notés après les traitements. On ne peut donc invoquer l'action du facteur pluie pour expliquer les chutes enregistrées.

Par ailleurs, la comparaison (tabl. 5) des captures faites dans le champ non traité d'Ury avec celles de $L_{1}$ et $L_{2}$ montre bien qu'à Ury, au cours des 3 semaines considérées (couvrant les dates d'application des produits), les niveaux de captures aussi bien en PP qu'en PC se maintiennent, alors qu'on note très nettement la chute après les traitements du 19 juin pour $\mathrm{L}_{1}$ et du 12 juin pour les $\mathrm{L}_{2}$. Il faut remarquer que la chute des captures en $\mathrm{L}_{1}$ et $\mathrm{L}_{2}$ survient avec une semaine d'écart qui correspond au décalage des traitements dans ces 2 champs.
Ces remarques nous permettent de dire avec certitude que les chutes de captures constatées sont bien dues aux interventions insecticides.

B) Mise en évidence des principales espèces affectées par les traitements

Pour réaliser ce type d'observation, nous avons opéré de 2 manières :

- d'une part, en plaçant au sol, le jour du traitement, 1 bâche en matière plastique d'environ $1 \mathrm{~m}^{2}$ par champ, destinće à recueillir les insectes tués,

- d'autre part, en analysant spécifiquement le matériel récolté par piégeage de façon à mettre en évidence les espèces ou groupes les plus touchés.

Lc tableau 6 indique les espèces tuées et recueillies après le traitement. Les nombres d'individus récoltés sont peu

TABLEAU 6

Liste des insectes morts récoltés sur les bâches un jour après le traitement

List of dead insects collected on sheet one day after treatment.

\begin{tabular}{|c|c|c|c|c|c|}
\hline \multirow[b]{3}{*}{ COLEOPTERES } & \multicolumn{2}{|c|}{ Champ } & \multirow[b]{3}{*}{ DIPTERES NEMATOCERES } & \multicolumn{2}{|c|}{ Champ } \\
\hline & $\mathrm{L}_{\mathrm{l}}$ & $\mathrm{L}_{2}$ & & $\mathbf{L}_{1}$ & $\mathrm{~L}_{2}$ \\
\hline & & & & & \\
\hline $\begin{array}{l}\text { Larves } \\
\text { Corticarina gibbosa }\end{array}$ & 1 & & Smittia & & 2 \\
\hline $\begin{array}{l}\text { Corticarina gibbosa } \\
\text { Atomaria linearis }\end{array}$ & & 1 & Cecidomyidac & 8 & 6 \\
\hline $\begin{array}{l}\text { Atomaria linearis } \\
\text { Enicmus transversus }\end{array}$ & & 2 & Sciaridac & 3 & 5 \\
\hline & & 1 & Mycctophilidae & 2 & \\
\hline $\begin{array}{l}\text { Cantharis lateralis } \\
\text { Elateridae divers }\end{array}$ & 1 & & Dilophus febrilis & & 3 \\
\hline & 1 & & DIPTËRES BRACHYCERES & & \\
\hline $\begin{array}{l}\text { Staphylinidae } \\
\text { Atheta triangulum }\end{array}$ & & & Lonchoptcra lutea & & 1 \\
\hline & & 3 & Phoridac & & 1 \\
\hline $\begin{array}{l}\text { Alcocharien } \\
\text { Tachyporus chrysomelinus }\end{array}$ & & 1 & Scatella stenhammari & & 1 \\
\hline $\begin{array}{l}\text { Tachyporus chrysomelinus } \\
\text { Oxytclus sculpturatus }\end{array}$ & & 5 & Parascaptomyza disticha & & 1 \\
\hline $\begin{array}{l}\text { Oxytclus sculpturatus } \\
\text { Oxytelinae }\end{array}$ & & 5 & Tachydromia sp. & & 1 \\
\hline $\begin{array}{l}\text { Oxytclinae } \\
\text { Tachyporinae }\end{array}$ & 2 & & Platychirus sp. & & 1 \\
\hline $\begin{array}{l}\text { Tachyporinae } \\
\text { Oxypoda brachyptera }\end{array}$ & 1 & & PUCERONS & & \\
\hline $\begin{array}{l}\text { Oxypoda brachyptera } \\
\text { Carabidae }\end{array}$ & 1 & & Mctopolophium dirhodum & 1 & 1 \\
\hline $\begin{array}{l}\text { Carabidae } \\
\text { Platysma vulgare }\end{array}$ & & & $\begin{array}{l}\text { Larves diverses } \\
\text { CICADELLES }\end{array}$ & 1 & $\begin{array}{l}1 \\
1\end{array}$ \\
\hline Trechus 4 striatus & $\begin{array}{r}24 \\
1\end{array}$ & & COLLEMBOLES & 13 & 213 \\
\hline THYSANOPTËRES & 9 & 5 & Chenille: Cnephasia pumicana & & 2 \\
\hline HYMENOPTËRES & 4 & 4 & Chenilles diverses & & 1 \\
\hline & & & ARACHNIDES & & 15 \\
\hline & & & ACARIENS & & 3 \\
\hline
\end{tabular}


élevés du fait de la faible importance des surfaces de récupération des bâches. Les tableaux 7 et 8 répertorient les variations des captures dans le temps mettant ainsi en évidence, par la baisse des niveaux de capture, les espèces ou groupes qui sont les plus affectés par le traitement.

Ces relevés montrent que l'action de l'insecticide a un effet différé, la baisse enregistrée n'étant sensible que 2 jours après la pulvérisation. En outre, on peut remarquer que ce sont les mêmes insectes que ceux trouvés morts sur les bâches. Cette concordance fait apparaître une bonne homogénéité des résultats.

\section{CONCLUSIONS}

L'ensemble des résultats accumulés au cours de ces 2 années d'étude nous renseigne avec précision sur l'incidence des traitements (essenticllement insecticides) sur les entomocénoses.

Les premiers points sur lesquels il faut attirer l'attention ressortent de l'observation des captures globales, aussi bien en 1978 qu'en 1979, entre La Chapelle-la-Reine et Ury en zone non traitée et Bretonvilliers en zone traitée.

Nous constatons des différences importantes entre les niveaux de capture en faveur des zones non traitées, pour les mêmes périodes de piégeage : 97061 à La Chapelle-LaReine contre 53711 à Bretonvilliers en 1978; 94132 à La Chapelle-La-Reine contre 12261 à Bretonvilliers en 1979.

Il y a une moins grande richesse en espèces en zone traitée.

Dans les effectifs capturés, les espèces nuisibles représentent un pourcentage plus important en zone traitée.

Ces faits nous semblent significatifs. Ils montrent que le processus de modification de l'entomofaune, à la suite des interventions phytosanitaires répétées, est engagé dans un sens défavorable pour les équilibres biocénotiques. Compte tenu de ces informations, on peut être inquiet sur ce qu'il risque d'advenir si le rythme des interventions est intensifié et surtout si ces interventions sont généralisées à l'ensemble des cultures céréalières en France. Ce risque est encore accru par l'utilisation de produits peu spécifiques à longue rémanence.

Un autre aspect important est mis en évidence par l'analyse des captures, avant et après les applications d'insecticides. En effet, il apparaît nettement, et ceci confirme l'hypothèse que nous avions émise dès 1975 , que les traitements phytosanitaires, en particulier insecticides, détruisent une «tranche de vie » et perturbent ainsi sérieusement l'équilibre déjà précaire des agrosystèmes céréaliers.

L'expérimentation de 1979 à Louville-La-Chenard montre clairement que les captures exprimées en moyennes journalières sont, après les traitements, inférieures de 60 à 80 p. 100 à ce qu'elles étaient auparavant.

Les populations d'espèces nuisibles (Sitobion avenae, Metopolophium dirhodum, Zyginidia scutellaris, Empoasca pteridis, Oscinella frit, ...) étaient extrêmement faibles aux époques des traitements (12 et 19 juin). Il en résulte que ce sont essentiellement des espèces auxiliaires (Carabidae, Staphylinidae, Hyménoptères, Arachnides ...) et beaucoup d'espèces détritiphages (Collemboles, Sciaridae, Cecidomyidae, Chironomidae ...) qui ont été détruites comme le montre notre étude.

Si on s'en tient strictement à l'impact des traitements sur l'entomofaune, on peut dire que les interventions en cours de végétation ont une incidence sévère sur les populations d'insectes dont la majeure partie peut être considérée comme utile au bon fonctionnement de l'agrosystème.

\section{TABLEAU 7}

Espèces (ou groupes) les plus affectés par le traitement. Traitement au diméthoate du 19.6.1979 (Champ $L_{l}$ ). Les données sont exprimées en captures journalières.

$I B$ : intérieur du champ, PP : pots pièges ; $P C$ : plateaux colorés.

Species (or groups) most affected by the treatment. Treatment with dimethoate on 19.VI.1979 (Field $L_{1}$ ). Data are given in daily catches. $I B$; inside the field, PP : pitfall traps; $P C$ : yellow traps.

\begin{tabular}{|c|c|c|c|c|c|c|c|c|c|c|c|}
\hline & & & & $\operatorname{dim}$ & hoate & & & & & & \\
\hline & 16.6 & 17.6 & 18.6 & 19.6 & 20.6 & 21.6 & 22.6 & 23.6 & 24.6 & 25.6 & \\
\hline $\begin{array}{l}\text { Total des captures } \\
\text { dont }\end{array}$ & 630 & 421 & 499 & 715 & 1000 & 575 & 376 & 246 & 244 & 208 & \\
\hline Collemboles & 518 & 315 & 236 & 555 & 838 & 501 & 327 & 229 & 192 & 150 & \\
\hline Arachnides & 9 & 24 & 12 & 26 & 14 & 10 & 7 & 2 & 26 & 27 & ספ ימי \\
\hline Acaricns & 23 & 9 & 13 & 20 & 66 & 40 & 4 & 2 & 1 & 6 & IB.PF \\
\hline Platysma vulgare & 53 & 29 & 139 & 76 & 28 & 0 & 3 & 2 & 2 & 6 & \\
\hline Staphylinidae & 8 & 15 & 21 & 23 & 19 & 5 & 10 & 1 & 15 & 10 & \\
\hline Hyménoptères & & 11 & 2 & 2 & 5 & 0 & 3 & 0 & 1 & 2 & \\
\hline $\begin{array}{l}\text { Total des captures } \\
\text { dont }\end{array}$ & 111 & 154 & 492 & 348 & 284 & 167 & 161 & 111 & 96 & 70 & \\
\hline Collemboles & 37 & 10 & 18 & 16 & 21 & 14 & 23 & 0 & 3 & 11 & \\
\hline Arachnides & 0 & 2 & 0 & 2 & 1 & 0 & 1 & 2 & 2 & 0 & \\
\hline Hyménoptères & 5 & 24 & 48 & 23 & 19 & 11 & 8 & 5 & 3 & 10 & \\
\hline Thysanoptères & 30 & 36 & 220 & 187 & 159 & 36 & 54 & 19 & 13 & 5 & IB.PC \\
\hline Nématocères & 26 & 37 & 55 & 51 & 31 & 25 & 31 & 29 & 8 & 21 & \\
\hline Delia platura & 6 & 17 & 27 & 5 & 2 & 0 & 0 & 1 & 0 & 0 & \\
\hline Hydrellia griseola & 2 & 5 & 42 & 10 & 1 & 0 & 0 & 8 & 28 & 1 & \\
\hline Phoridac & 0 & 6 & 33 & 20 & 13 & 16 & 12 & 6 & 9 & 4 & \\
\hline Pucerons & 0 & 1 & 0 & 0 & 8 & 8 & 8 & 4 & 3 & 0 & \\
\hline
\end{tabular}




\section{TABLEAU 8}

Espèces (ou groupes) les plus affectées par le traitement. Traitement avec phosalone + parathion méthyl du 12.6.1979 (Champ L2). Les données sont exprimées en captures journalières. IB : intérieur du champ; $P P$ : pots pièges; $P C$ : plateaux colorés.

Species (or groups) most affected by the treatment. Treatment with phosalone + methyl parathion on 12.VI.1979 (Field $\left.L_{2}\right)$. Data are given in daily catches. $I B:$ inside the field ; PP : pitfall traps ; $P C:$ yellow traps.

\begin{tabular}{|c|c|c|c|c|c|c|c|c|c|c|c|}
\hline & & phos & ne $+p$ & athion & éthyl & & & & & & \\
\hline & 7.6 & 11.6 & 12.6 & 13.6 & 14.6 & 15.6 & 16.6 & 17.6 & 18.6 & 19.6 & \\
\hline $\begin{array}{l}\text { Duréc du pićgcage } \\
\text { en jour }\end{array}$ & 4 & 4 & 1 & 1 & 1 & 1 & 1 & 1 & 1 & 1 & \\
\hline $\begin{array}{l}\text { Total capturé } \\
\text { journalier } \\
\text { dont }\end{array}$ & 860 & 965 & 687 & 711 & 184 & 63 & 94 & 59 & 123 & 141 & \\
\hline Collemboles & 815 & 906 & 614 & 648 & 150 & 36 & 44 & 30 & 61 & 98 & \\
\hline Arachnides & 4 & 8 & 10 & 12 & 13 & 15 & 23 & 12 & 11 & 15 & \\
\hline Acariens & & 2 & 2 & & 4 & 1 & 9 & 3 & 25 & 3 & IB.PP \\
\hline Platysma vulgare & 2 & 5 & 8 & 5 & 2 & 2 & 0 & 5 & 9 & 8 & \\
\hline Anchomenus dorsalis & 1 & 5 & 1 & 1 & 0 & 0 & 0 & 1 & 0 & 3 & \\
\hline Oxypoda brachyptera & 12 & 6 & 9 & 4 & 0 & 0 & 0 & 0 & 0 & 1 & \\
\hline Aleocharien & 7 & 5 & 9 & 4 & 0 & 0 & 1 & 0 & 4 & 0 & \\
\hline Hyménoptères & 1 & 1 & 1 & 2 & 0 & 0 & 0 & 1 & 1 & 1 & \\
\hline $\begin{array}{l}\text { Total capturé } \\
\text { journalier } \\
\text { dont }\end{array}$ & 674 & 522 & 321 & 374 & 101 & 51 & 90 & 44 & 207 & 53 & \\
\hline Collemboles & 429 & 359 & 129 & 184 & 8 & 8 & 4 & 1 & 3 & 0 & \\
\hline Arachnides & 2 & 2 & 7 & 4 & 3 & 3 & 0 & 5 & 3 & 2 & \\
\hline Meligethes acneus & 55 & 5 & 4 & 24 & 0 & 0 & 0 & 1 & 8 & 4 & \\
\hline Staphylinidae & 2 & 3 & 5 & 6 & 0 & 0 & 0 & 0 & 2 & 1 & \\
\hline Hyménoptères & 30 & 14 & 19 & 8 & 8 & 4 & 4 & 9 & 16 & 7 & IB.PC \\
\hline Thysanoptères & 14 & 20 & 0 & 29 & 0 & 0 & 1 & 0 & 0 & 3 & \\
\hline Nématocères & 30 & 76 & 50 & 23 & 15 & 5 & 15 & 9 & 12 & 10 & \\
\hline Delia platura & 9 & 1 & 2 & 1 & 5 & 3 & 5 & 4 & 29 & 3 & \\
\hline Phoridac & 36 & 15 & 9 & 51 & 7 & 0 & 2 & 0 & 27 & 17 & \\
\hline Hydrellia griscola & 1 & 1 & 3 & 7 & 6 & 6 & 2 & 5 & 7 & 1 & \\
\hline Puccrons & 7 & 1 & 9 & 0 & 10 & 2 & 1 & 0 & 18 & 5 & \\
\hline
\end{tabular}

\section{CONCLUSIONS GÉNÉRALES ET DISCUSSION}

Les recherches entreprises depuis 1974 sur les biocénoses céréalières montrent que dans ces cultures évoluent les populations de nombreuses espèces (de 800 à 1000 ) dont la plupart jouent un rôle bénéfique pour le fonctionnement de ces agrosystc̀mes.

En ce qui concerne les espèces nuisibles actuellement présentes, nous avons pu mettre en évidence, au cours de ces 7 années, que les rotations courtes maïs-blé ne sont pas plus défavorables que celles incluant une dicotylédone (betterave-pois) tous les 2 ans. En définitive, ce sont essenticllement les conditions climatiques de l'hiver et de la période de végétation qui déterminent les niveaux de population des ravagcurs (CHAMBON, 1982).

Dans un autre ordre d'idéc, l'expérimentation réalisée pour connaitre l'incidence des traitements insecticides sur la faune a démontré leur action secondaire néfaste aux équilibres biocénotiques. D'unc part, dans les régions traitées intensément depuis plusieurs années, les niveaux de populations sont très bas mais les espèces nuisibles occupent une place prépondérante. D'autre part, l'analyse fine du matériel récolté montre un effet de choc des interventions sur l'entomofaune, les captures, après un traitement insecticide. accusant des chutes de l'ordre de 70 à 80 p. 100.

Les espèces les plus touchées, compte tenu de la composi- tion de la faune sont, de toute évidence, des espèces utiles au sens le plus large du terme.

La recolonisation des champs par une entomofaune diversifiée qui est constatée actuellement n'est possible que grâce à la faible proportion des surfaces traitées. Une évolution, dans le sens de l'intensification et la généralisation des interventions avec des produits peu spécifiques ne pourrait qu'aggraver la situation, par l'accélération du processus déjà engagé.

Si on considère maintenant l'état sanitaire des cultures céréalières en ce qui concerne les ravageurs, il apparaît que nous ne sommes pas encore en situation extrême comme cela s'cst produit par le passé en arboriculture fruitière. En zone céréalière traitéc, nous n'avons pas jusqu'alors observé (sauf quelques cas isolés) de situation dégradée au point d'ĉtre à l'origine de pullulations anormales de ravageurs. Rappelons qu'en 1979 la forte pullulation de $M$. tirhodum dont les conséquences se sont fait sentir sur maïs, a été généralisée touchant aussi bien les zones traitées que zelles non traitées.

Cette situation est duc en grande partic au fait que la cadence actuelle des traitements et les surfaces concernécs n'ont pas encore atteint un niveau d'irréversibilité et permettent encore le maintien de réserves d'auxiliaires et une possibilité de recolonisation.

Néanmoins, cu égard aux tendances qui se dégagent de 
nos investigations, un grand effort de vulgarisation doit être fait pour assurer le développement de la protection intégrée en grande culture afin d'enrayer le processus de dégradation déjà amorcé.

Si le contraire se produisait, on assisterait rapidement à un appauvrissement du patrimoine faunistique en faveur des nuisibles, exacerbant les problèmes de ravageurs non seulement en céréaliculture mais aussi pour d'autres productions végétales, comme le font remarquer très justement ViCKERMANN \& SUNDERLAND (1977).

Pour se convaincre, si besoin est, de la gravité de ce risque, rappelons les pullulations de $R$. padi sur maïs en 1980 notées après des interventions irréfléchies contre la pyrale (Ostrinia nubilalis $\mathrm{Hb}$.) ayant éliminé les auxiliaires aphidiphages. Si dans ce cas précis il est possible d'établir la relation directe destruction des aphidiphages $\rightarrow$ pullulation de pucerons, nous ne sommes pas à même, dans l'état actuel des connaissances sur la biologie des insectes, de déterminer avec précision les conséquences de la disparition d'espèces dont le rôle dans la régulation des populations de nuisibles ou pour le bon fonctionnement de l'agrosystème céréalier n'est pas encore bien connu. Cependant, nous pouvons émettre comme hypothèse vraiscmblable que les répercussions d'un tel phénomène seront à coup sûr néfastes à plus ou moins long terme.
Pour élargir la portée de ces investigations, il faut aussi attirer l'attention sur le fait que la raréfaction des insectes de mi-juin à mi-juillet peut avoir des conséquences non négligeables pour certains éléments de la faune cynégétique. A titre d'exemple, rappelons que la perdrix grise, dont les poussins éclosent à cette période, se nourrissent presque exclusivement d'insectes au cours des 2 premières semaines de leur vie.

Reçu le 11 juin 1981. Accepté le 14 décembre 1981.

\section{RÉFÉRENCES BIBLIOGRAPHIQUES}

Chambon J. P., 1975. Labiocénose et la lutte contre les ravageurs des céréales. Déf. des Vég., ${ }^{\circ}$ 176, 1-10.

Chambon J. P., 1982. Recherches sur les biocénoses céréalières. I. Incidence à long terme des rotations mais-blé sur les niveaux de population d'insectes ravageurs. Agronomie, 2, 4 (sous presse).

Vickerman G. P., Sunderland K. D., 1977. Some effects of dimethoatc on arthropods in winter wheat. J. appl. Ecol. 14, 767777. 